\title{
Reference values for cardiopulmonary exercise testing in healthy volunteers:
} the SHIP study

\author{
B. Koch*,+, C. Schäper*,+, T. Ittermann\#, T. Spielhagen*, M. Dörr*, H. Völzke ${ }^{\#,}$ \\ C.F. Opitz , R. Ewert* and S. Gläser*
}

ABSTRACT: Cardiopulmonary exercise testing (CPET) is a widely applied clinical procedure. The aim of the present study was to acquire a comprehensive set of reference values for cardiopulmonary responses to exercise and to evaluate possible associations with sex, age and body mass index (BMI).

A standardised progressive incremental exercise protocol on a cycle ergometer was applied to 1,708 volunteers of a cross-sectional epidemiologic survey, called "Study of Health in Pomerania". Individuals with cardiopulmonary disorders, or echocardiographic or lung function pathologies, were excluded. The influence of potential confounding factors, such as smoking, taking $\beta$-blockers, hypertension, diastolic dysfunction, BMI and physical activity, were analysed for their influencing power. Reference values of CPET parameters were determined by regression analyses.

Of the volunteers, 542 current smokers and obese individuals were excluded for not being representative of a healthy population. The final sample size was 534 (253 males), with age 2580 yrs. The current study provides a representative set of reference values for CPET parameters based on age and weight.

Sex and age have a significant influence on exercise parameters. While addressing the problem of a selection bias, the current study provides the first comprehensive set of reference values obtained in a large number of healthy volunteers within a population-based survey.

KEYWORDS: Cardiopulmonary exercise testing, oxygen uptake, reference values, Study of Health in Pomerania, ventilatory efficiency

ardiopulmonary exercise testing (CPET) is a widely applied routine procedure in daily clinical work, used for the investigation of cardiac and pulmonary disorders. Due to their prognostic and therapeutic implications [1, 2], the normalcy of response to incremental exercise is usually considered with respect to particular functional indices, such as the peak oxygen uptake $\left(V^{\prime} \mathrm{O}_{2}\right.$,peak), the estimated lactate threshold $(\theta \mathrm{L})$ and the characterisation of ventilatory efficiency expressed by the minute ventilation $\left(V^{\prime} \mathrm{E}\right)$ changes as a function of the pulmonary carbon dioxide output ( $V^{\prime} \mathrm{E}-V^{\prime} \mathrm{CO}_{2}$ slope). Reference values for these indices have been established, usually with respect to sex, age, body features and physical activity, by several investigators [3-5]. The interpretation of the results of exercise tests requires knowledge of the normal response [6], but a consensus has not been reached on the definition of normalcy. The commonly used reference values are based on exercise capacities of shipyard workers [3] or university members [4]. "Healthy volunteers" were usually defined as subjects without self-reported evidence or clinical symptoms and signs of heart and lung disease, a normal resting electrocardiogram (ECG), a normal 12-lead ECG response to an exercise stress test and the absence of any medication, especially any with cardiorespiratory effects. However, obtainable reference data sets show relevant limitations, considering their sample recruitment, age distribution and the type of exercise protocol, as well as the lack of consideration of echocardiographic and lung function data.

The aim of the present study was to acquire a comprehensive set of reference values on gas exchange and exercise capacity in cardiopulmonary exercise in a representative, healthy population. To minimise selection bias for the study
AFFILIATIONS

*Dept of Cardiology, Pneumology and Infectious Diseases,

\#Institute for Community Medicine, SHIP/Clinical-Epidemiological

Research, University of Greifswald, Greifswald,

"Dept of Cardiology, DRK-Kliniken Berlin/Westend, Berlin, Germany. ${ }^{+}$Both authors contributed equally to this article.

CORRESPONDENCE

B. Koch

Medical Faculty of the Ernst-MoritzArndt University, Dept of Pulmonary Medicine and Infectious Diseases,

Friedrich-Loeffler-Str. 23, D-17475 Greifswald, Germany.

Fax: 4938348622422

E-mail: beate.koch@

uni-greifswald.de

Received:

May 152008

Accepted after revision:

August 202008

SUPPORT STATEMENT

This work is part of the Community Medicine Research net (CMR) of the University of Greifswald (Greifswald, Germany), which is funded by grants from the German Federal Ministry of Education and Research (BMBF, Berlin, Germany; grants 01ZZ0403, $01 Z Z 0103$ and 01GI0883), the Ministry for Education, Research and Cultural Affairs and the Ministry of Social Affairs of the Federal State of MecklenburgWest Pomerania (both Schwerin, Germany). The CMR encompasses several research projects that share population-based data from the Study of Health in Pomerania.

STATEMENT OF INTEREST None declared.

European Respiratory Journal Print ISSN 0903-1936 Online ISSN 1399-3003 
population, a standardised progressive incremental exercise protocol on a cycle ergometer was performed by healthy subjects recruited from a large-scale, cross-sectional, population-based cohort in north-east Germany.

\section{SUBJECTS AND METHODS}

\section{Study population}

Study volunteers consisted of participants of the Study of Health in Pomerania (SHIP). SHIP is a cross-sectional, population-based survey in a region in the north-east of Germany. The study details are given elsewhere [7]. In brief, from the entire study population of 212,157 inhabitants living in the area, a sample was selected from the population registration offices, where all German inhabitants are registered. A two-stage cluster sampling method was adopted from the World Health Organization (WHO) Monitoring Cardiovascular Disease Project in Augsburg, Germany [8]. A representative sample was drawn, comprising 7,008 adults aged 20-79 yrs with 292 persons of each sex in each of the twelve 5-yr age strata. The net sample (without migrated or deceased people) consisted of 6,267 eligible subjects, of whom 4,310 individuals eventually participated in the baseline study of SHIP (SHIP-0). Data collection started in October 1997 and finished in March 2001. From March 2003 until July 2006, a 5-yr follow-up examination was performed (SHIP-1). The sample (without migrated, deceased or nonresponding people) then comprised 3,300 subjects (1,589 males and 1,711 females) aged 25-85 yrs. Of those, 1,708 individuals (834 males and 874 females) volunteered for a standardised progressive incremental exercise test on a cycle ergometer.

All participants were investigated in health examination centres established for the purpose of the study and gave written informed consent. The study conformed to the principles of the Declaration of Helsinki as reflected by an $a$ priori approval of the Ethics Committee of the University of Greifswald (Greifswald, Germany).

\section{Pre-exercise diagnostics and exclusion criteria}

Socio-demographic and behavioural characteristics, as well as information on medical histories, were assessed using computerassisted personal interviews, which were administered by trained and certified staff. The previous history of diseases was based on self-reported physician's diagnosis. The definition of cardiopulmonary disorders was based on self-reported physician's diagnosis, use of specific medication (based on the anatomic, therapeutic and chemical (ATC) code) [9], electro- or echocardiographical pathological findings, and lung function abnormalities measured by spirometry and body plethysmography. Normal lung function was defined according to the recommendations of the European Coal and Steel Community [10]. All clinical tests were performed by experienced, trained and certified physicians. All examiners were trained in an experienced clinical department before entering the study. Initial certification was awarded to potential observers after a minimum of 3 months of training. Observers were held to strict quality criteria. To facilitate comparability between SHIP and other population-based studies in Germany, external observers were regularly invited to participate in certification procedures. The data collection phase was monitored by a Data Safety and Monitoring Committee (Greifswald).
Height and weight were measured for the calculation of the body mass index (BMI; body weight in $\mathrm{kg}$ divided by square of height in metres). Systolic and diastolic blood pressure were measured three times in seated subjects after a 5-min rest period, with each reading being followed by an additional rest period of $3 \mathrm{~min}$. Mean blood pressure was calculated from the last two measurements. A 12-lead ECG was taken of each participant. The medication was recorded by a computer-aided method using the ATC code. A blood sample was drawn from the cubital vein in the supine position.

Subjects with the following criteria were excluded from the present study: past myocardial infarction, signs of ischaemia/ infarction, right or left bundle branch block or WolffParkinson-White syndrome in the ECG; the presence of a cardiac pacemaker, stenosis or insufficiency of the cardiac valves, systolic and diastolic failures or a cardiac shunt vitium found in the echocardiography; pulmonary diseases, bronchial asthma, chronic obstructive bronchitis (ratio of forced expiratory volume over one second (FEV1) to forced vital capacity being $<0.7$ and the FEV1 being $<80 \%$ predicted [11]) or emphysema (intrathoracic gas volume being $>140 \%$ predicted, residual volume percentage of total lung capacity being $>140 \%$ predicted), all defined by spirometric/body-plethysmographic criteria and/or patients' self report; neuromuscular or musculoskeletal disorders based on neurological examination; anaemia and/or (with the exception of $\beta$-blockers) the use of drugs with influence on cardiopulmonary function. Patients with a breathing reserve ( $V^{\prime} \mathrm{E} /$ maximum voluntary ventilation (MVV)) $>0.8 \%$ were also excluded from the study.

\section{Exercise testing}

A symptom-limited exercise test using one calibrated electromagnetically braked cycle ergometer with an electrical seat height adjustment (Ergoselect 100; Ergoline, Bitz, Germany) was performed according to a protocol modified from JoNES et al. [4] (stepwise increase in work load of $16 \mathrm{~W} \cdot \mathrm{min}^{-1}$, starting with unloaded cycling plus the ergometer-related permanent load). Gas exchange and ventilatory variables were analysed breath by breath using a computer-based system. Each operation was preceded by a resting period of $\geqslant 3 \mathrm{~min}$ to reach steady-state conditions. Steady-state status was analysed for oxygen uptake $\left(V^{\prime} \mathrm{O}_{2} ; \mathrm{mL} \cdot \mathrm{min}^{-1}\right), V^{\prime} \mathrm{CO}_{2}\left(\mathrm{~mL} \cdot \mathrm{min}^{-1}\right), V^{\prime} \mathrm{E}$ $\left(\mathrm{L} \cdot \mathrm{min}^{-1}\right)$, ventilatory equivalents for oxygen and carbon dioxide $\left(V^{\prime} \mathrm{E} / V^{\prime} \mathrm{O}_{2}\right.$ and $V^{\prime} \mathrm{E} / V^{\prime} \mathrm{CO}_{2}$, respectively) and end-tidal gas tensions for oxygen and carbon dioxide $(\mathrm{mmHg})$.

The procedure was continuously monitored by a physician. In the absence of chest pain and ECG abnormalities, all tests were continued as symptom-limited (volitional exertion, dyspnoea or fatigue). Prior to the test, patients were encouraged to reach maximal exhaustion, while during exercise no further motivational interventions were utilised.

All tests were performed in room air according to current guidelines for exercise testing, with continuous monitoring of ECG, blood pressure and oxygen saturation [12, 13].

\section{Gas exchange variables}

Respiratory gas exchange variables were measured continuously throughout the resting period, the unloaded cycling period and the exercise test, using a VIASYS Healthcare system 
(Oxycon Pro or Rudolph's mask; VIASYS GmbH, Hoechberg, Germany). Prior to each test, the equipment was calibrated in standard fashion with reference gas and volume calibration. A standard 12-lead ECG was obtained at rest, at every minute during exercise, and for $\geqslant 5 \mathrm{~min}$ during recovery; blood pressure was measured with a standard cuff sphygmomanometer. $V^{\prime} \mathrm{E}\left(\mathrm{L} \cdot \mathrm{min}^{-1}\right)$, tidal volume $(V \mathrm{~T}$; in litres $), V^{\prime} \mathrm{O}_{2}$ and $V^{\prime} \mathrm{CO}_{2}$ (both $\mathrm{mL} \cdot \mathrm{min}^{-1}$ ) were acquired on a breath-by-breath basis and averaged over 10-s intervals. $V^{\prime} \mathrm{O}_{2}$,peak was defined as the highest 10 -s average of $V^{\prime} \mathrm{O}_{2}$ in the last minute of exercise. The $V^{\prime} \mathrm{E} / V^{\prime} \mathrm{CO}_{2}$ was assessed at rest, at $\theta \mathrm{L}$ and at peak exercise. In addition, the slope of the regression of both parameters ( $V^{\prime} \mathrm{E}-V^{\prime} \mathrm{CO}_{2}$ slope), excluding excess hyperventilation at the end of exercise, was assessed [2]. The $\theta \mathrm{L}$ was determined according to WASSERMAN et al. [6]. The $V^{\prime} \mathrm{E} / \mathrm{MVV}$ was calculated as peak $V^{\prime} \mathrm{E}$ in relation to MVV. MVV was calculated as the FEV1 multiplied by 41 [14].

\section{Statistical analysis}

The data were analysed separately for each age and sex stratum. The age strata were graded into five groups: 1) 25-34, 2) $35-44,3) 45-54,4) 55-64$ and 5) $\geqslant 64$ yrs of age.

Logistic regression analyses were performed to compare the participants of cycle ergometry with the entire SHIP-1 cohort, in order to detect any selection bias. ANOVA adjusted to sex and age was performed in order to evaluate the influence of specific confounding factors on selected parameters, such as $V^{\prime} \mathrm{O}_{2}$,peak and $V^{\prime} \mathrm{O}_{2}$ at the $\theta \mathrm{L}$, both adjusted for weight. ANOVA was performed on both the absolute values of $V^{\prime} \mathrm{O}_{2}$,peak and $V^{\prime} \mathrm{O}_{2}$ at $\theta \mathrm{L}\left(\mathrm{mL} \cdot \mathrm{min}^{-1}\right)$ and the body weight-corrected results $\left(\mathrm{mL} \cdot \mathrm{min}^{-1} \cdot \mathrm{kg}^{-1}\right)$.

For the evaluation of reference values, quantile regression analysis, a statistical method for estimating models for the conditional quantile functions [15], adjusted for age, sex and BMI, was performed. Since quantile regression makes no distributional assumption, an initial transformation of the original data was unnecessary.

Statistical significance was defined as $p<0.01$. Values are given as median and 5 th and 95th percentiles. The participants were divided into a normal weight (BMI $\leqslant 25 \mathrm{~kg} \cdot \mathrm{m}^{-2}$ ) and an overweight group (BMI $>25$ but $<30 \mathrm{~kg} \cdot \mathrm{m}^{-2}$ ).

\section{Confounding factors}

The following variables were statistically examined for a possible impact on exercise parameters. 1) Smoking habits, classified as nonsmoker, current smoker or former smoker. 2) $\beta$-blocker medication. 3) Hypertension at rest, defined according to the WHO standards as systolic blood pressure $\geqslant 140 \mathrm{mmHg}$ or diastolic blood pressure $\geqslant 90 \mathrm{mmHg}$ at rest, or self-reported use of antihypertensive medications [16]. 4) Diastolic dysfunction, defined as an early to late diastolic filling $(\mathrm{E} / \mathrm{A})$ ratio $<1$ (for those aged $\leqslant 50 \mathrm{yrs}$ ) or $<0.5$ (for those aged $>50 \mathrm{yrs}$ ) and no systolic dysfunction [17]. 5) BMI, classified as BMI $<19 \mathrm{~kg} \cdot \mathrm{m}^{-2}$ (underweight), BMI 19$25 \mathrm{~kg} \cdot \mathrm{m}^{-2}$ (normal weight), BMI $25-30 \mathrm{~kg} \cdot \mathrm{m}^{-2}$ (overweight) or $\mathrm{BMI}>30 \mathrm{~kg} \cdot \mathrm{m}^{-2}$ (obesity), defined based on the current WHO classification. 6) Exercise habits, classified as $<1$ h weekly, 2-3 h weekly, 3-4 h weekly and $>4$ h weekly.

\section{RESULTS}

\section{Selection of the reference population}

Relevant characteristics and distributions of all study participants are shown in table 1 . The mean $\pm S D$ age of the participants was $52.02 \pm 13.57$ yrs

\section{Confounding factors on the CPET parameters}

The following factors were examined by ANOVA, adjusted for both sex and age, for their influence on $V^{\prime} \mathrm{O}_{2}$,peak and $V^{\prime} \mathrm{O}_{2}$ at $\theta \mathrm{L}$ (as absolute values and corrected for body weight).

According to their smoking habits, a significant difference was found between the current smokers and the former smokers as well as the nonsmokers. There was no difference found between the non- and former smokers. Consequently, current smokers were excluded from the study. The $\beta$-blocker use did not prove to be significant for any of the variables. Therefore, all participants with $\beta$-blocker medication were included in the study. Arterial hypertension did not prove to have a significant influence on the selected CPET parameters; thus, hypertensive participants were included. No significant influence on the selected parameters was found concerning diastolic dysfunction. Therefore, this was not an exclusion criterion. Obese participants (BMI $>30 \mathrm{~kg} \cdot \mathrm{m}^{-2}$ ) had to be excluded from the study, since a significant influence on the CPET parameters was found when comparing normal to obese volunteers for all

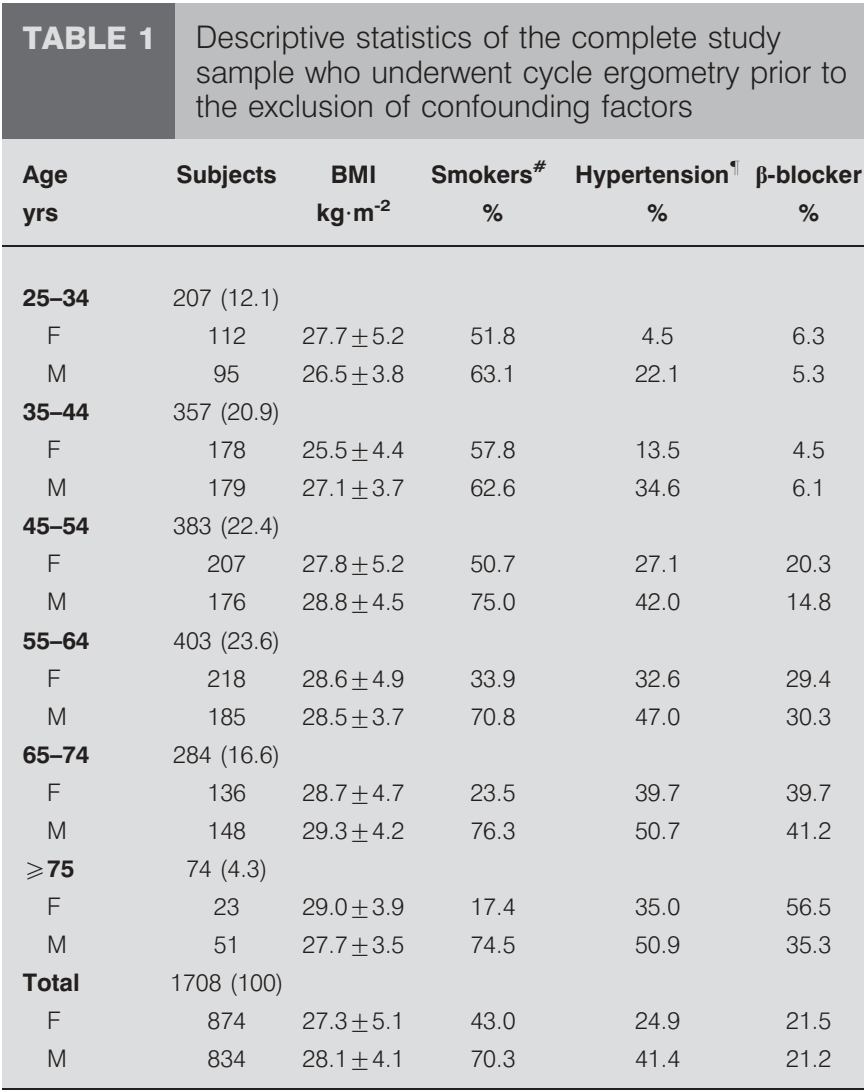

Data are presented as $n(\%), n$ or mean $\pm \mathrm{SD}$, unless otherwise stated. BMI body mass index; F: female; M: male. \# : current smokers; " : defined according to the World Health Organization standards as systolic blood pressure $\geqslant 140 \mathrm{mmHg}$ or diastolic blood pressure $\geqslant 90 \mathrm{mmHg}$ at rest 


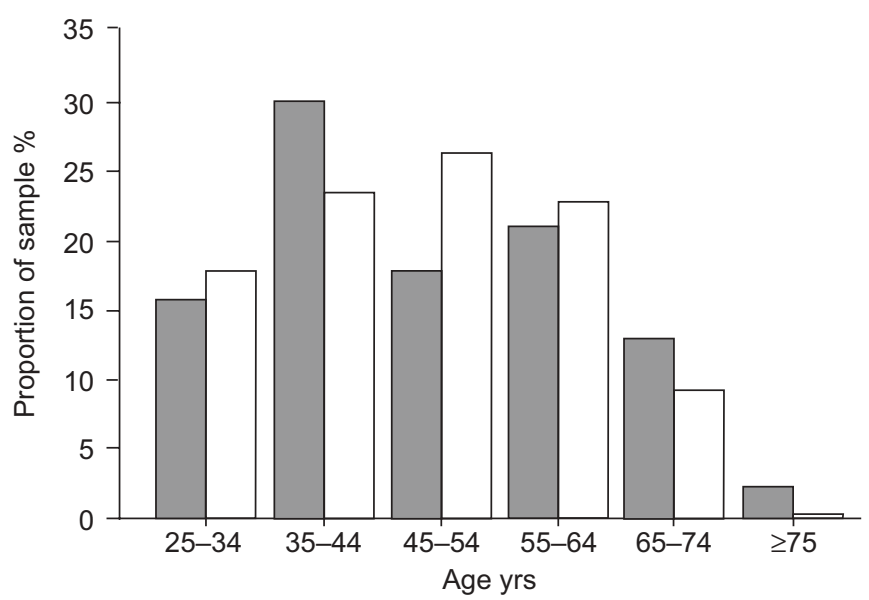

FIGURE 1. Distribution of the study sample subdivided into 10-yr age categories. $\square$ : males, $\mathrm{n}=253$; $\square$ : females, $\mathrm{n}=281$.

variables. The influence of physical activity was not consistently significant throughout the investigated groups. Therefore, physical activity was not an exclusion criterion.

In conclusion, current smokers $(n=265)$, obese participants $(n=225)$ and obese smokers $(n=52)$ were excluded from the study sample. In total, 632 individuals were excluded for medical reasons. The final sample size was 534 (253 male and 281 female) of the initial 1,708 participants in CPET. The age distribution of the study sample is shown in figure 1.

\section{Comparison between the complete SHIP-1 population and CPET participants}

A significant difference $(p=0.025)$ was found between the participants and the nonparticipants in CPET, with more nonsmoking volunteers participating in CPET. Also, there were significantly fewer hypertensive people among the participants in CPET $(\mathrm{p}<0.01)$. No significant difference was found between the participants in CPET and the nonparticipants, with regard to BMI status and the occurrence of pulmonary diseases $(p=0.14)$.

\section{Exercise protocol}

The main reasons for termination of exercise in the present study were muscular exertion and fatigue $(72.9 \%)$, as well as dyspnoea $(27.1 \%)$. The mean exercise duration of the present study sample, subdivided into $10-y r$ age categories, was $\sim 11 \mathrm{~min}$ in the 25-34-yr age group and $7.8 \mathrm{~min}$ for the individuals aged $>65$ yrs (table 2 ).

\section{Reference values}

$\mathrm{V}^{\prime} \mathrm{O}_{2}$, peak and $\mathrm{V}^{\prime} \mathrm{O}_{2}$ at $\theta \mathrm{L}$

In the investigated study sample, $V^{\prime} \mathrm{O}_{2}$, peak revealed a significant dependency on age, sex and BMI. $V^{\prime} \mathrm{O}_{2}$, peak declined with advancing age and increasing BMI, and was lower for females in comparison with males (fig. 2). The effect of age, sex and BMI can be calculated by a predictive equation, as shown in table 3 .

In the male population with a $\mathrm{BMI}$ of $\leqslant 25 \mathrm{~kg} \cdot \mathrm{m}^{-2}, V^{\prime} \mathrm{O}_{2}$ at $\theta \mathrm{L}$ averaged $54 \%$ (5th percentile) and $60 \%$ (95th percentile) of $V^{\prime} \mathrm{O}_{2}$,peak. In those with a BMI of $>25 \mathrm{~kg} \cdot \mathrm{m}^{-2}$ and $<30 \mathrm{~kg} \cdot \mathrm{m}^{-2}$

\begin{tabular}{lcc} 
TABLE 2 & $\begin{array}{l}\text { Exercise durations subdivided into } \\
\text { categories }\end{array}$ & 10-yr age \\
Age yrs & Exercise duration min & Subjects $\mathbf{~}$ \\
\hline $\mathbf{2 5 - 3 4}$ & $11.02 \pm 3.36$ & 92 \\
$\mathbf{3 5 - 4 4}$ & $11.29 \pm 3.43$ & 145 \\
$\mathbf{4 5 - 5 4}$ & $9.31 \pm 2.86$ & 115 \\
$\mathbf{5 5 - 6 4}$ & $9.08 \pm 2.73$ & 120 \\
$\mathbf{6 5 - 7 4}$ & $7.81 \pm 2.23$ & 55 \\
$\geqslant \mathbf{7 5}$ & $7.98 \pm 1.16$ & 7 \\
Total & $9.92 \pm 3.24$ & 534 \\
\hline &
\end{tabular}

the $V^{\prime} \mathrm{O}_{2}$ at $\theta \mathrm{L}$ averaged $52 \%$ (5th percentile) and 64\% (95th percentile) of $V^{\prime} \mathrm{O}_{2}$,peak. In the female population with a BMI of $\leqslant 25 \mathrm{~kg} \cdot \mathrm{m}^{-2}$ the $V^{\prime} \mathrm{O}_{2}$ at $\theta \mathrm{L}$ was $55 \%$ (5th percentile) and $62 \%$ (95th percentile) of $V^{\prime} \mathrm{O}_{2}$, peak. Females with a BMI of $>25 \mathrm{~kg} \cdot \mathrm{m}^{-2}$ and $<30 \mathrm{~kg} \cdot \mathrm{m}^{-2}$ had an average $V^{\prime} \mathrm{O}_{2}$ at $\theta \mathrm{L}$ of $53 \%$ (5th percentile) and $62 \%$ (95th percentile) of $V^{\prime} \mathrm{O}_{2}$,peak.
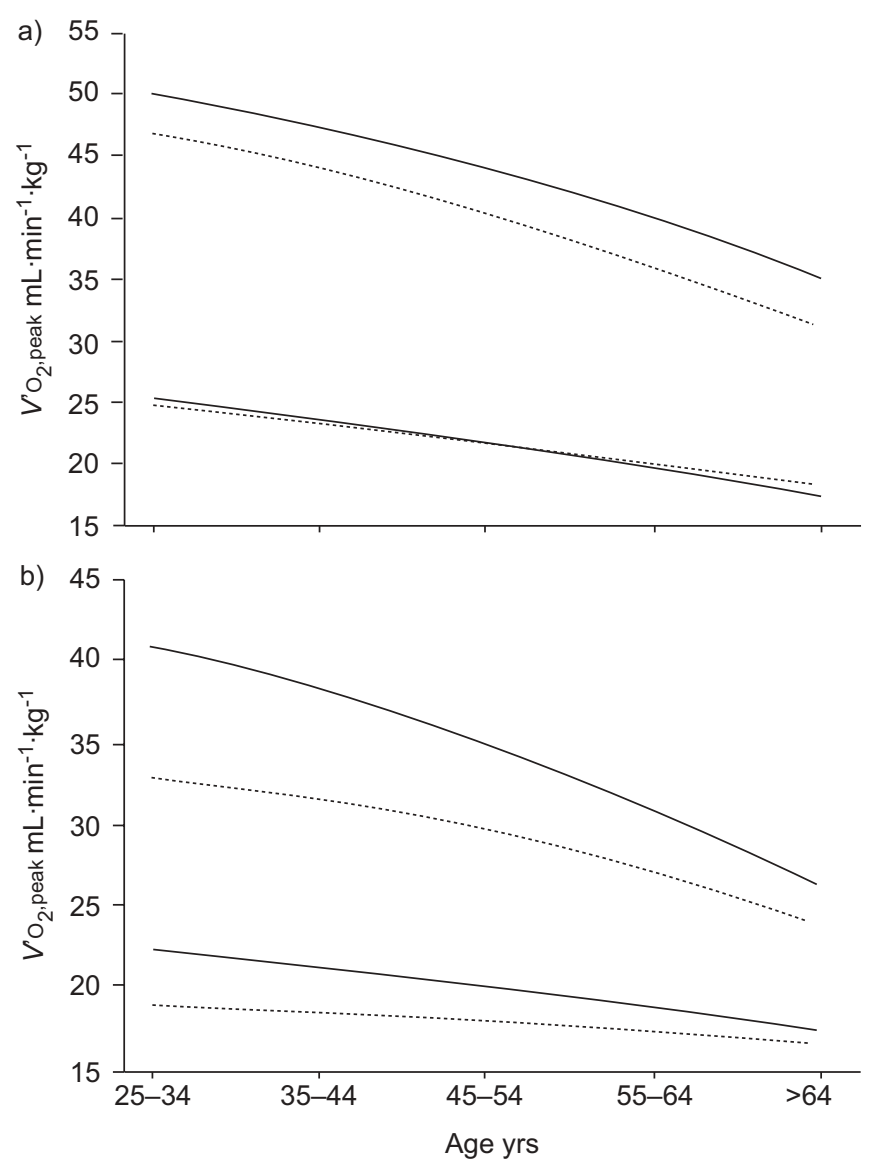

FIGURE 2. Normal ranges for peak oxygen uptake $\left(V^{\prime} \mathrm{O}_{2}\right.$, peak $)$ for a) males and b) females, with relation to age and subdivided according to body mass index (BMI) groups. - : BMl $\leqslant 25 \mathrm{~kg} \cdot \mathrm{m}^{-2} ; \cdots \cdot$ : BMl $>25 \mathrm{~kg} \cdot \mathrm{m}^{-2}$. Lower and upper lines represent the 5 th and 95 th percentiles, respectively. 


\begin{tabular}{|c|c|c|c|c|c|c|c|c|c|}
\hline & Intercept & Age & $\mathrm{Age}^{2}$ & Sex & BMI & Age $\cdot B M I$ & Sex·BMI & Age.sex & Age $\cdot$ sex $\cdot B M I$ \\
\hline \multicolumn{10}{|c|}{$V^{\prime} \mathrm{O}_{2}$,peak } \\
\hline \multicolumn{10}{|c|}{$\mathrm{mL} \cdot \mathrm{min}^{-1} \cdot \mathbf{k g}^{-1}$} \\
\hline $5 \%$ & 30.9643 & -2.5661 & -0.0263 & -3.7224 & 1.8765 & 0.1082 & -2.9703 & 0.7361 & 0.2799 \\
\hline \multicolumn{10}{|c|}{$\begin{array}{l}V^{\prime} \mathrm{O}_{2} \text { at } \theta \mathrm{L} \\
\mathrm{mL} \cdot \mathrm{min}^{-1} \cdot \mathrm{kg}^{-1}\end{array}$} \\
\hline $5 \%$ & 8.0266 & 2.1582 & -0.2337 & 0.9685 & -0.9729 & 0.4670 & -0.5278 & -0.5030 & -0.1150 \\
\hline Median & 18.3219 & 0.3399 & -0.0369 & -1.1117 & 1.5436 & -0.9756 & -1.8656 & -0.2643 & 0.5895 \\
\hline $95 \%$ & 31.4007 & -0.4891 & 0.0681 & -2.7167 & 12.5034 & -3.4748 & -7.0634 & -0.7041 & 1.5570 \\
\hline
\end{tabular}

Data are regression coefficients for each variable obtained by quantile regression. For entering subjects' characteristics, sex was coded as 1 for males and 2 for females. Body mass index (BMI) was coded as 0 for BMI $\leqslant 25 \mathrm{~kg} \cdot \mathrm{m}^{-2}$ and 1 for BMI $>25 \mathrm{~kg} \cdot \mathrm{m}^{-2}$. There were five age groups: $25-34,35-44,45-54,55-64$ and $\geqslant 64 \mathrm{yrs}$ of age. The content of each column was multiplied with the coded characteristics. To calculate the $5 \%$, median and $95 \%$ reference ranges, the contents of each column have to be added

Although there was a wide range of $\left(V^{\prime} \mathrm{O}_{2}\right.$ at $\left.\theta \mathrm{L}\right) /\left(V^{\prime} \mathrm{O}_{2}\right.$,peak $)$ percentage within the age groups, no value $<41 \%$ was found. Within both sexes, there was no significant dependency of $V^{\prime} \mathrm{O}_{2}$ at $\theta \mathrm{L}$ on age or BMI, particularly according to the 5th percentile, whereas there seemed to be an age-correlated decline within the 95th percentile. This correlation is shown in figure 3. The effect of age, sex and BMI can be calculated by a predictive equation, as shown in table 3.

\section{$V^{\prime} E / V^{\prime} \mathrm{CO}_{2}$ at rest and $\theta \mathrm{L}$ and $V^{\prime} E-V^{\prime} \mathrm{CO}_{2}$ slope}

The mean $\pm \mathrm{SD} V^{\prime} \mathrm{E} / V^{\prime} \mathrm{CO}_{2}$ at rest was $27.7 \pm 0.7$ and $42.3 \pm 1.2$ for the 5th and 95th percentiles, respectively, in males, versus $26.7 \pm 0.64$ and $39 \pm 1.6$ in females. With advanced age, an increase among the reference values was found. No value $>50$ was observed in either males or females. The corresponding equation can be found in table 4 .

The mean $\pm \mathrm{SD} V^{\prime} \mathrm{E} / V^{\prime} \mathrm{CO}_{2}$ at $\theta \mathrm{L}$ was $22.3 \pm 0.6$ and $30.6 \pm 0.8$ for the 5 th and 95th percentiles, respectively, in males, versus $22.3 \pm 0.52$ and $31.8 \pm 1.2$ in females. With advancing age, an increase among the reference values was found. No value $>35$ was observed in either males or females. The corresponding equation can be found in table 4 .

Ventilatory efficiency during exercise did not differ with respect to sex. In contrast, an age-correlated increase within the reference values was found. For males, the mean $\pm \mathrm{SD}$ $V^{\prime} \mathrm{E}-V^{\prime} \mathrm{CO}_{2}$ slope increased from $18.00 \pm 0.9$ and $30.00 \pm 1.50$ for percentiles 2.5 and 97.5, respectively, at mean (range) age 29 (25-34) yrs, to $21.00 \pm 1.30$ and $34.00 \pm 3.15$ for percentiles 2.5 and 97.5 , respectively, at age $>64$ yrs (fig. 4). Aside from the differences in the standard deviations, similar values were found for females. The corresponding equation can be found in table 4.

\section{Further ventilatory results}

Calculations for additional reference values, such as respiratory frequency, $V^{\prime} \mathrm{E}, V \mathrm{~T}, V^{\prime} \mathrm{E} / \mathrm{MVV}$, oxygen pulse $\left(V^{\prime} \mathrm{O}_{2} /\right.$ cardiac frequency), and end-tidal carbon dioxide tension are shown in table 4 , for the oxygen pulse depicted in table 3.

\section{DISCUSSION}

The present study provides the first set of reference values for CPET compiled for a representative sample of healthy volunteers using the platform of a population-based study, the SHIP population. By excluding subjects with known cardiopulmonary disorders or certain cardiovascular risk factors, a disease-free population was selected.

Most previous studies of reference values for cardiopulmonary exercise variables in healthy individuals have been based on small sample sizes and differed significantly with respect to the populations studied and the methods used [3-6, 18-20]. For example, the reference values established by WASSERMAN et al. [6] are based on data obtained from 77 male shipyard workers with a mean age of $54 \mathrm{yrs}$, including some who were smokers, obese or hypertensive. They were judged to have normal cardiorespiratory systems based on medical history, physical examination, ECG during rest and exercise, chest radiograph, pulmonary function test and exercise performance. However, objective parameters, such as echocardiographic or bodyplethysmographic findings, were not taken into account. JONES et al. [4] recruited their participants from the local university and the general population by advertising. These individuals were stratified into five age groups for both sexes until a total sample size of 50 for each sex was reached. "Health" was defined by gaining information on medical history, leisure and occupational habits, exercise-related symptoms and pulmonary function tests. Athletes, subjects 

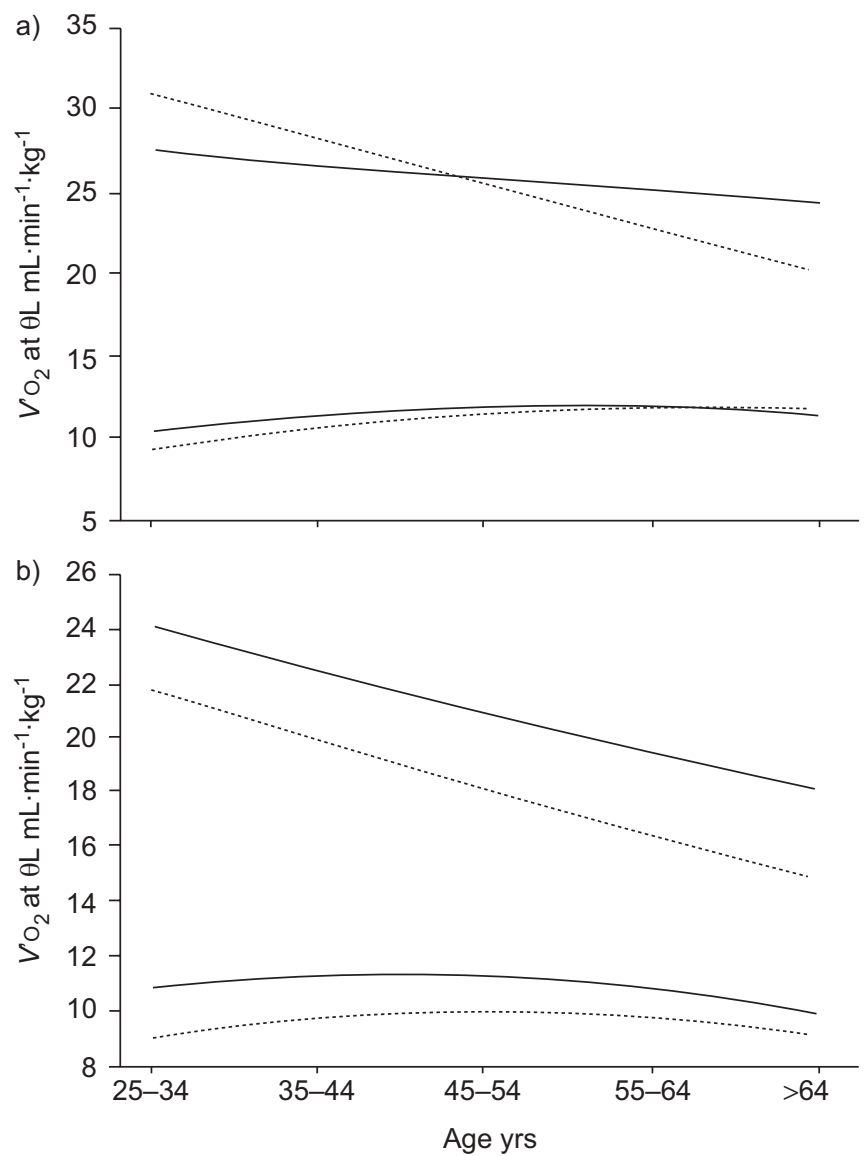

FIGURE 3. Normal ranges for oxygen uptake $\left(V^{\prime} \mathrm{O}_{2}\right)$ at lactate threshold $(\theta \mathrm{L})$ for a) males and b) females, with relation to age and subdivided according to body mass index (BMI) groups. _- BMl $\leqslant 25 \mathrm{~kg} \cdot \mathrm{m}^{-2} ; \cdots \cdot . \mathrm{BMl}>25 \mathrm{~kg} \cdot \mathrm{m}^{-2}$. Lower and upper lines represent the 5th and 95th percentiles, respectively.

with serious illness in the past or any chronic disorders, as well as individuals suffering from hypertension, were excluded. Smokers remained within the study sample. INBAR et al. [19] established reference values for cardiopulmonary responses in 1,424 males aged 20-70 yrs, who underwent an incremental treadmill exercise. Individuals with abnormal ECG tracings, medical history, or physical or laboratory findings of cardiac, respiratory, metabolic or neuromuscular diseases were excluded from the study. Current or former smokers were included. Again, no echocardiographic and body-plethysmographic investigation was performed. Reference values were only provided for male volunteers; recruitment methods were not stated.

Statistical results based on these previous studies are, however, less likely to provide a good estimate of population parameters. Therefore, the contribution made by the present study lies in the selection of a quantifiably healthy population, involving several types of clinical examination methods, including echocardiography, pulmonary function tests and body plethysmography, as well as information gained from the medical history. These rigorous inclusion criteria are responsible for the fact that only 534 of the initial 1,708 individuals were includable. Furthermore, the investigation of confounding factors was of importance for the precision of reference values.

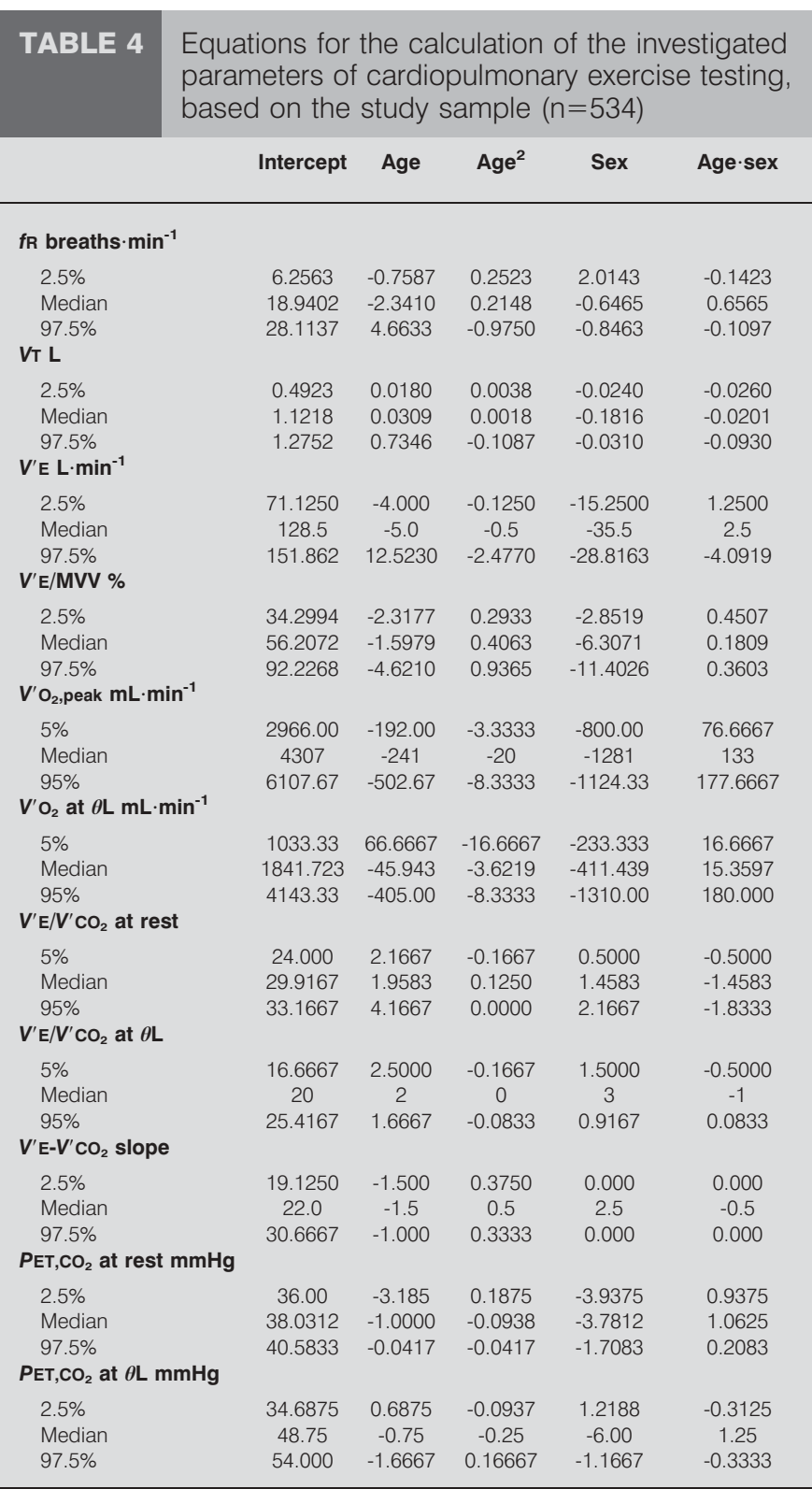

Data are regression coefficients for each variable obtained by quantile regression. Fo entering subjects' characteristics, sex was coded as 1 for males and 2 for females. There were five age groups: $25-34,35-44,45-54,55-64$ and $\geqslant 64$ yrs of age. The content of each column was multiplied with the coded characteristics. To calculate the $2.5 \%, 5 \%$, median, $95 \%$ and $97.5 \%$ reference ranges, the contents of each column have to be added. $\mathrm{fR}$ : respiratory frequency; $V T$ : tidal volume; $V^{\prime} E$ : minute ventilation $V^{\prime} E / M V V$ : breathing reserve; $V^{\prime} \mathrm{O}_{2}$, peak: peak oxygen uptake; $V^{\prime} \mathrm{O}_{2}$ : oxygen uptake; $\theta \mathrm{L}$ lactate threshold; $V^{\prime} \mathrm{E} / V^{\prime} \mathrm{CO}_{2}$ : ventilatory equivalent for carbon dioxide; $V^{\prime} \mathrm{E}-V^{\prime} \mathrm{CO}_{2}$ slope: ventilatory efficiency expressed by the $V^{\prime} E$ changes as a function of the pulmonary carbon dioxide output; $P$ ET, $\mathrm{CO}_{2}$ : end-tidal carbon dioxide tension.

Concerning the exercise mode, it should be mentioned that the $V^{\prime} \mathrm{O}_{2}$,peak measured during treadmill exercise is about $10 \%$ higher than that obtained via cycle ergometry [18]. Therefore, the reference values presented in the current study should only be applied to cycling tests.

The advantage of a population-based study lies in the general applicability of the collected data. Nevertheless, reference values are statistical markers of the value of distribution of a specific test. Thus, factors influencing the distribution of these 

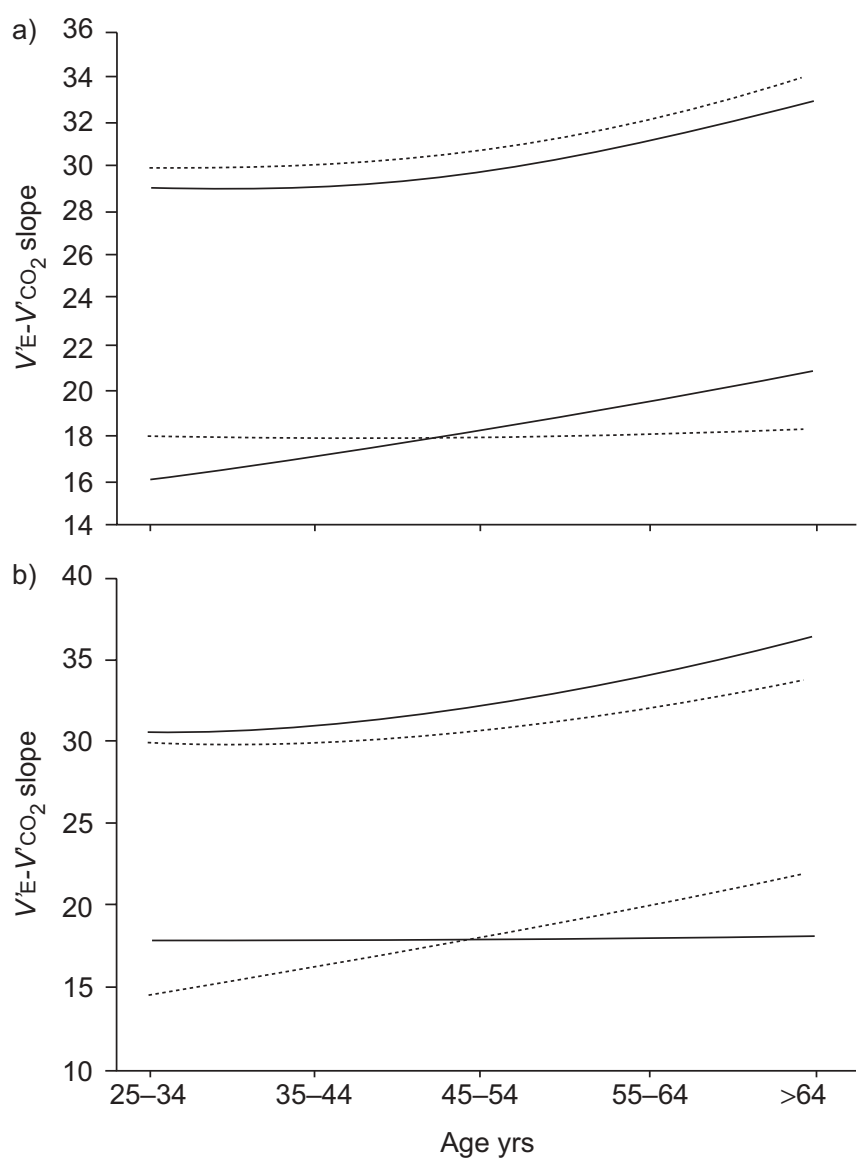

FIGURE 4. Normal ranges for ventilatory efficiency expressed by the minute ventilation $\left(V^{\prime} E\right)$ changes as a function of the pulmonary carbon dioxide output $\left(V^{\prime} \mathrm{E}-V^{\prime} \mathrm{CO}_{2}\right.$ slope) for a) males and b) females, with relation to age and subdivided according to body mass index (BMl) groups. —: BMl $\leqslant 25 \mathrm{~kg} \cdot \mathrm{m}^{-2} ; \cdots \cdot$ : BMl $>25 \mathrm{~kg} \cdot \mathrm{m}^{-2}$. Lower and upper lines represent the 5 th and 95 th percentiles, respectively.

values may also affect reference values and should be taken into account.

\section{Oxygen uptake}

The main results of the present study suggest that it is important to include sex, age and BMI when predicting $V^{\prime} \mathrm{O}_{2}$,peak. Previous population-based studies of $V^{\prime} \mathrm{O}_{2}$,peak in Europe [18, 20] and North America [21, 22] reported remarkably uniform data, despite different study samples and testing protocols.

Using the equation established in the present study, the values for a male aged 35-44 yrs with a BMI of $\leqslant 25 \mathrm{~kg} \cdot \mathrm{m}^{-2}$ were 23.47 and $47.27 \mathrm{~mL} \cdot \mathrm{min}^{-1} \cdot \mathrm{kg}^{-1}$ (5th and 95th percentiles, respectively). For a female of similar age and BMI, the values were 21.22 and $38.29 \mathrm{~mL} \cdot \mathrm{min}^{-1} \cdot \mathrm{kg}^{-1}$ (5th and 95th percentiles, respectively). The $V^{\prime} \mathrm{O}_{2}$,peak predicted by WASSERMAN et al. [6] for a 40-yr-old male with a BMI of $24.22 \mathrm{~kg} \cdot \mathrm{m}^{-2}$ was $36.76 \mathrm{~mL} \cdot \mathrm{min}^{-1} \cdot \mathrm{kg}^{-1}$ and for a female of the same age and $\mathrm{BMI}$ this was $25.47 \mathrm{~mL} \cdot \mathrm{min}^{-1} \cdot \mathrm{kg}^{-1}$. The $V^{\prime} \mathrm{O}_{2}$, peak predicted by

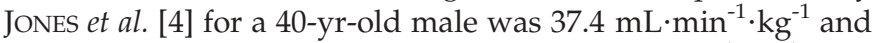
for a female of the same age it was $28.6 \mathrm{~mL} \cdot \mathrm{min}^{-1} \cdot \mathrm{kg}^{-1}$. The
$V^{\prime} \mathrm{O}_{2}$,peak predicted by NEDER et al. [23] for a 40-yr-old male of height $170 \mathrm{~cm}$ and weight $70 \mathrm{~kg}$ was $32.4 \mathrm{~mL} \cdot \mathrm{min}^{-1} \cdot \mathrm{kg}^{-1}$ and for a female of the same age, height and weight it was $24.91 \mathrm{~mL} \cdot \mathrm{min}^{-1} \cdot \mathrm{kg}^{-1}$.

As presented in figure 5, it is obvious that the values for $V^{\prime} \mathrm{O}_{2}$,peak found in the literature lie within the 5th-95th percentile range of the current study. For female subjects especially, a linear approximation towards the 5 th percentile is obvious. This might be due to the selection criteria applied, or the age distribution in the cited studies. By comparing the median values established from the present study with those found in literature, a tendency towards higher values in individuals aged $>45$ yrs becomes apparent in both sexes. This, the current authors assume, is due primarily to age distribution within the investigated studies and secondarily to cohort effects due to changes in individual, behavioural and environmental factors over decades.

The relevance and importance of the $\theta \mathrm{L}$ for the characterisation of cardiovascular and metabolic abnormalities was established by WASSERMAN and MCILROY [24] and, later, by WEBER and JANICKI [25]. In the present study, gas exchange criteria were used in order to identify the $\theta \mathrm{L}$. The current data show a steady decline of the $\theta \mathrm{L}$ with advancing age. When calculated as the ratio of $\left(V^{\prime} \mathrm{O}_{2}\right.$ at $\left.\theta \mathrm{L}\right) /\left(V^{\prime} \mathrm{O}_{2}\right.$,peak $)$, values between 52 and $64 \%$ were found. The lowest values were found in the youngest age group, whereas the highest values emerged in the oldest age group. Thus, it can be postulated that the $\theta \mathrm{L}$ increases as a percentage of $V^{\prime} \mathrm{O}_{2}$,peak with advancing age $[3,5]$. JONES et al. [4] and DAVIS et al. [26] have also studied the effect of age on the $\theta \mathrm{L}$. Although different $\theta \mathrm{L}$ detection methods were applied, both groups found the absolute $\theta \mathrm{L}$ declining with age in both sexes, but less than the decrease in $V^{\prime} \mathrm{O}_{2}$,peak. The ratio of $\left(V^{\prime} \mathrm{O}_{2}\right.$ at $\left.\theta \mathrm{L}\right) /\left(V^{\prime} \mathrm{O}_{2}\right.$,peak $)$ tends to increase with age and tends to be higher in females than males. These findings are in agreement with the data of the present study.

\section{Ventilatory equivalent}

Ventilation during exercise is primarily associated with metabolic needs and is therefore closely related to $V^{\prime} \mathrm{CO}_{2}[19]$ In order to assess the efficiency of $V^{\prime} \mathrm{E}$ with respect to carbon dioxide elimination during exercise, the $V^{\prime} \mathrm{E} / V^{\prime} \mathrm{CO}_{2}$ was used. The present study demonstrated that the ventilatory response to carbon dioxide production during exercise increased with advancing age. The $V^{\prime} \mathrm{E}-V^{\prime} \mathrm{CO}_{2}$ slope was significantly correlated to age for both sexes. The mean $\pm \mathrm{SD}$ value from previous studies was $31 \pm 4.5$ for $V^{\prime} \mathrm{E} / V^{\prime} \mathrm{CO}_{2}[19,27]$; this value is comparable to those found in the current study sample.

SUlLIVAN et al. [28] found the $V^{\prime} \mathrm{E} / V^{\prime} \mathrm{CO}_{2}$ at rest to be $\sim 36$ and to decrease with exercise to 30. HANSEN et al. [3] reported that, among 77 males with mean age 54 yrs, they found a $V^{\prime} \mathrm{E} / V^{\prime} \mathrm{CO}_{2}$ of $39 \pm 10$ at rest and of $29 \pm 4$ at $\theta \mathrm{L}$. These values are similar to those found in the current study sample and lie within the 5th95th percentile range of the present data.

With respect to the CPET protocol applied in the present study, it should be emphasised that variations in incremental step size during CPET can significantly affect exercise time and maximal power. In daily practice, protocols according to the recommendations of WASSERMAN et al. [6] are widely used to reach exercise durations between 6 and $12 \mathrm{~min}$. Recent recommendations for 

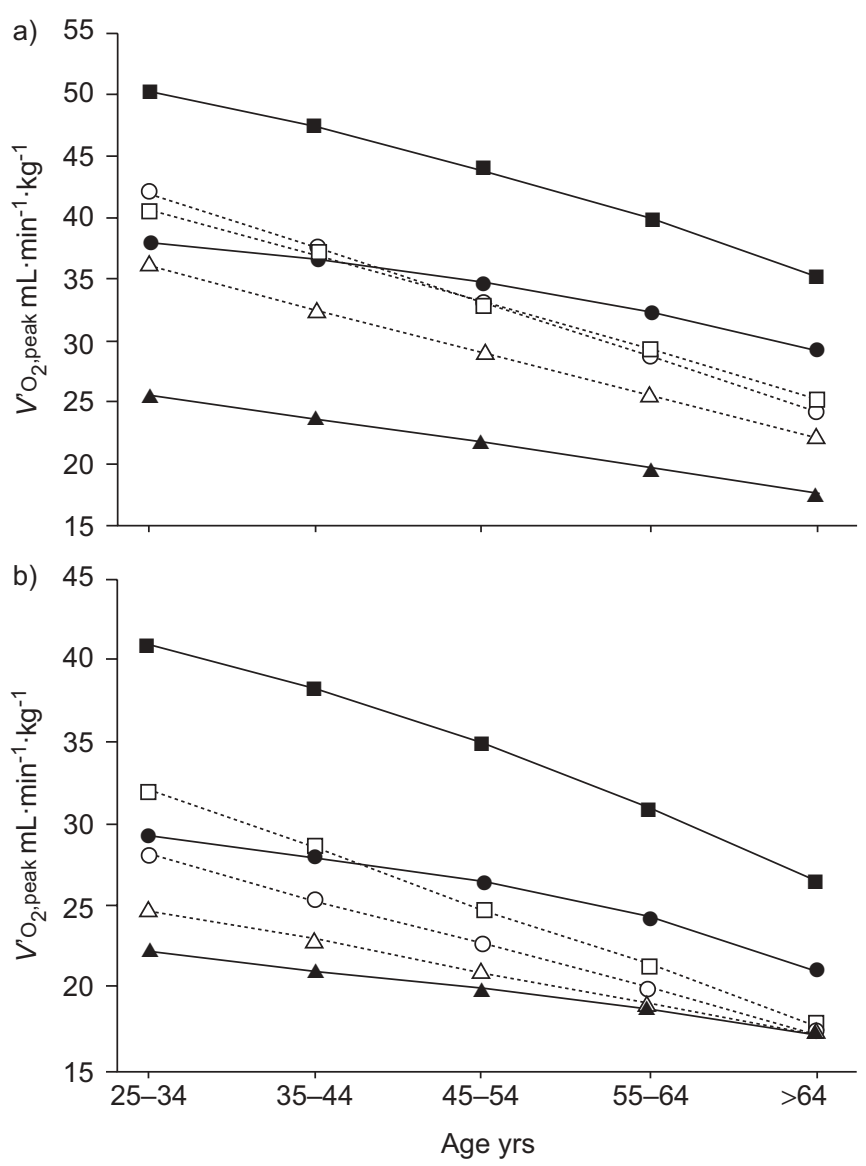

FIGURE 5. Peak oxygen uptake $\left(\mathrm{V}^{\prime} \mathrm{O}_{2}\right.$,peak $)$ subdivided into 10-yr age categories for a) males and b) females. In each case, a comparison with historical data was performed for an exemplary individual (height $170 \mathrm{~cm}$, weight $70 \mathrm{~kg}$ ). —: current study data; $\cdots \cdot$.. historical data. $\mathbf{\square}:$ 95th percentile; $\boldsymbol{\bullet}$ : median; $\mathbf{\Lambda}$ : 5th percentile; $\square$ : data from Jones et al. [4]; $\bigcirc$ : data from WASSERMAN et al. [6]; $\triangle$ : data from NEDER et al. [23].

exercise protocol in healthy volunteers are based on relatively small numbers of subjects [29-31], but emphasise that the duration of exercise should be $8-17 \mathrm{~min}$, as appropriate. Other parameters of importance for clinical judgement and prognosis, such as $V^{\prime} \mathrm{O}_{2}$, exercise ventilation and ventilatory efficiency, appear to be less dependent on variations in exercise protocol. Therefore, an individualisation of incremental step size to reach maximal results for the parameters aforementioned does not appear to be mandatory [32].

In order to assess the generalisability of the present study results, population-specific factors, including the relatively high mean BMI $\left(27.4\right.$ and $26.8 \mathrm{~kg} \cdot \mathrm{m}^{-2}$ in males and females, respectively) and the prevalence of cardiopulmonary disorders, have to be taken into account [33]. A high prevalence of hypertension is found in West Pomerania [34]. The current study may be criticised for not being multicentric and, therefore, may be considered to be limited in its applicability outside Germany. However, as the British Framingham study has shown, obesity and cardiovascular diseases are growing challenges in the entire western world [35]. The incidence of obesity and nicotine abuse in the current study is comparable to those reported in cohorts of other investigations. Therefore, the current authors assume that the reference values herein presented are applicable to similar study samples found in the western world.

In addition, the present study may be criticised for having a potential selection bias towards including younger, healthier and nonsmoking subjects, because participation in cycle ergometry was voluntary. However, since the collected values were adjusted for age, sex and BMI and tested against possible confounding factors, the differences between the general German population, the entire SHIP population and the participants in the CPET group were negligible.

Since obesity seems to be a major concern and growing challenge worldwide, further investigation on normal values of CPET for obese individuals is necessary.

\section{Conclusion}

In summary, the current study details the cardiopulmonary responses to incremental cycle ergometry exercise tests in normal, healthy subjects. The relatively wide ranges of response patterns indicate that individual responses vary widely. This finding has to be taken into account when analysing CPET data. Additionally, the results demonstrate that sex, age and anthropometric characteristics should be considered in the assessment of normalcy of dynamic exercise responses.

The results of the present study have been presented in several ways, partly to allow the investigator to compare studies in other laboratories with those of the present study and to encourage a flexible approach to the interpretation of exercise test results in general. The major strength of the current study lies in the use of data from a large population-based sample of adults and the guideline of excluding subjects with any known relevant diseases. Another relevant contribution lies in the weighting of different confounding factors on exercise performance and in the effort that was made to include several medical methods to establish a healthy study sample.

\section{REFERENCES}

1 Gitt AK, Wasserman K, Kilkowski C, et al. Exercise anaerobic threshold and ventilatory efficiency identify heart failure patients for high risk of early death. Circulation 2002; 106: 3079-3084.

2 Kleber FX, Vietzke G, Wernecke KD, et al. Impairment of ventilatory efficiency in heart failure: prognostic impact. Circulation 2000; 101: 2803-2809.

3 Hansen JE, Sue DY, Wasserman K. Predicted values for clinical exercise testing. Am Rev Respir Dis 1984; 129: S49-S55.

4 Jones NL, Makrides L, Hitchcock C, Chypchar T, McCartney N. Normal standards for an incremental progressive cycle ergometer test. Am Rev Respir Dis 1985; 131: 700-708.

5 Sue DY, Hansen JE. Normal values in adults during exercise testing. Clin Chest Med 1984; 5: 89-98.

6 Wasserman K, Hansen JE, Sue DY, Stringer WW, Whipp BJ. Principles of Exercise Testing and Interpretation: Including 
Pathophysiology and Clinical Applications. 4th Edn. Philadelphia, Lippincott Williams and Wilkins, 2004.

7 John U, Greiner B, Hensel E, et al. Study of Health In Pomerania (SHIP): a health examination survey in an east German region: objectives and design. Soz Praventivmed 2001; 46: 186-194.

8 Keil U, Stieber J, Döring A, et al. The cardiovascular risk factor profile in the study area Augsburg. Results from the first MONICA survey 1984/85. Acta Med Scand Suppl 1988; 728: 119-128.

9 Friedrich N, Alte D, Völzke H, et al. Reference ranges of serum IGF-1 and IGFBP-3 levels in a general adult population: results of the Study of Health in Pomerania (SHIP). Growth Horm IGF Res 2008; 18: 228-237.

10 Quanjer PH, Tammeling GJ, Cotes JE, Pedersen OF, Peslin R, Yernault JC. Lung volumes and forced ventilatory flows. Report Working Party Standardization of Lung Function Tests, European Community for Steel and Coal. Official Statement of the European Respiratory Society. Eur Respir J 1993; 6: Suppl. 16, 5-40.

11 Devereux G. ABC of chronic obstructive pulmonary disease. Definition, epidemiology, and risk factors. BMJ 2006; 332: 1142-1144.

12 Ross RM. ATS/ACCP statement on cardiopulmonary exercise testing. Am J Respir Crit Care Med 2003; 167: 1451.

13 ERS Task Force, Palange P, Ward SA, et al. Recommendations on the use of exercise testing in clinical practice. Eur Respir J 2007; 29: 185-209.

14 Miller WF, Scacci R, Gast LR. Laboratory Evaluation of Pulmonary Function. Philadelphia, Lippincott Williams and Wilkins, 1987.

15 Koenker R. Quantile Regression. Economic Society Monographs. New York, Cambridge University Press, 2005.

16 Whitworth JA, World Health Organization, International Society of Hypertension Writing Group. 2003 World Health Organization (WHO)/International Society of Hypertension (ISH) statement on management of hypertension. J Hypertens 2003; 21: 1983-1992.

17 Fischer M, Baessler A, Hense HW, et al. Prevalence of left ventricular diastolic dysfunction in the community. Results from a Doppler echocardiographic-based survey of a population sample. Eur Heart J 2003; 24: 320-328.

18 Lange Anderson K, Shephard RJ, Denolin H, Varnauskas E, Masironi R. Fundamentals of exercise testing. Geneva, World Health Organization, 1971.

19 Inbar O, Oren A, Scheinowitz M, Rotstein A, Dlin R, Casaburi R. Normal cardiopulmonary responses during incremental exercise in 20- to 70-yr-old men. Med Sci Sports Exerc 1994; 26: 538-546.

20 Astrand I. Aerobic work capacity in men and women with special reference to age. Acta Physiol Scand Suppl 1960; 49: $1-92$.
21 Drinkwater BL, Horvath SM, Wells CL. Aerobic power of females, ages 10 to 68. J Gerontol 1975; 30: 385-394.

22 Dehn MM, Bruce RA. Longitudinal variations in maximal oxygen intake with age and activity. J Appl Physiol 1972; 33: 805-807.

23 Neder JA, Nery LE, Castelo A, et al. Prediction of metabolic and cardiopulmonary responses to maximum cycle ergometry: a randomised study. Eur Respir J 1999; 14: 13041313.

24 Wasserman K, McIlroy MB. Detecting the threshold of anaerobic metabolism in cardiac patients during exercise. Am J Cardiol 1964; 14: 844-852.

25 Weber KT, Janicki JS. Cardiopulmonary Exercise Testing: Physiologic Principles and Clinical Applications. Philadelphia, Saunders, 1986; pp. 201-218.

26 Davis JA, Storer TW, Caiozzo VJ. Prediction of normal values for lactate threshold estimated by gas exchange in men and women. Eur J Appl Physiol Occup Physiol 1997; 76: 157-164.

27 Blackie SP, Fairbarn MS, McElvaney NG, Wilcox PG, Morrison NJ, Pardy RL. Normal values and ranges for ventilation and breathing pattern at maximal exercise. Chest 1991; 100: 136-142.

28 Sullivan MJ, Higginbotham MB, Cobb FR. Increased exercise ventilation in patients with chronic heart failure: intact ventilatory control despite hemodynamic and pulmonary abnormalities. Circulation 1988; 77: 552-559.

29 Buchfuhrer MJ, Hansen JE, Robinson TE, Sue DY, Wasserman K, Whipp BJ. Optimizing the exercise protocol for cardiopulmonary assessment. J Appl Physiol 1983; 55: 1558-1564.

30 Zhang YY, Johnson MC 2nd, Chow N, Wasserman K., Effect of exercise testing protocol on parameters of aerobic function. Med Sci Sports Exerc 1991; 23: 625-630.

31 Bentley DJ, McNaughton LR. Comparison of W(peak), $\mathrm{VO}_{2}$ (peak) and the ventilation threshold from two different incremental exercise tests: relationship to endurance performance. J Sci Med Sport 2003; 6: 422-435.

32 Gläser S, Lodziewski S, Koch B, Opitz CF, Völzke H, Ewert R. Influence of the incremental step size in work rate on exercise response and gas exchange in patients with pulmonary hypertension. BMC Pulm Med 2008; 8: 3.

33 Völzke H, Alte D, Neuhauser $\mathrm{H}$, et al. Risikopopulation Vorpommern. [Risk population Pomerania]. Ärztebl MecklVorpom 2007; 17: 49-53.

34 Meisinger C, Heier M, Völzke H, et al. Regional disparities of hypertension prevalence and management within Germany. J Hypertens 2006; 24: 293-299.

35 Foster MC, Hwang SJ, Larson MG, et al. Overweight, obesity, and the development of stage 3 CKD: the Framingham Heart Study. Am J Kidney Dis 2008; 52: 39-48. 